\title{
Primary Leptomeningeal Melanoma of the Cervical Spine Mimicking a Meningioma-A Case Report
}

\author{
Sascha Marx ${ }^{1}$ Steffen K. Fleck ${ }^{1} \quad$ Jotham Manwaring ${ }^{2} \quad$ Silke Vogelgesang $^{3}$ Soenke Langner ${ }^{4}$ \\ Henry W.S. Schroeder ${ }^{1}$
}

${ }^{1}$ Department of Neurosurgery, University of Greifswald, Sauerbruchstraße, Greifswald, Germany

2 Department of Neurosurgery and Brain Repair, University of South

Address for correspondence Sascha Marx, MD, Department of Florida, Tampa, Florida Neurosurgery, University of Greifswald, Sauerbruchstraße, 17491

${ }^{3}$ Department of Neuropathology, University of Greifswald, Greifswald, Germany

${ }^{4}$ Institut of Radiology and Neuroradiology, University of Greifswald, Sauerbruchstraße, Greifswald, Germany

J Neurol Surg Rep 2014;75:e93-e97.

\begin{abstract}
Background and Importance Primary leptomeningeal melanoma (PLM) is highly malignant and exceedingly rare. Due to its rarity, diagnostic and treatment paradigms have been slow to evolve. We report the first case of a PLM that mimics a cervical spine meningioma and then discuss the current clinical, radiologic, and pathologic diagnostic methodologies as well as expected outcomes related to this disease.

Clinical Presentation A 54-year-old woman presented a dural-based extramedullary solid mass ventral to the $\mathrm{C} 2-\mathrm{C} 3$ spinal cord causing spinal cord compression without cord signal changes, characteristic of meningioma. Intraoperative microscopic inspection revealed numerous black spots littering the surface of the dura; the tumor itself was yellow in appearance and had a soft consistency. Pathologic analysis of the specimen revealed a

Keywords

- CSF analysis

- histology

- meningioma

- misdiagnosis

- MRI characteristics

- primary leptomeningeal melanoma malignant melanin-containing tumor. No primary site was found, so a diagnosis of primary leptomeningeal melanoma was made, and the patient subsequently received interferon therapy. To date (2 years postoperatively), no local or systemic recurrence of the tumor has been identified.

Conclusion As with most rare tumors, case reports constitute the vast majority of references to PLM. Only an increased awareness and an extensive report of each individual case can help diagnose and clarify the nature of PLM. Clinicians need to be aware of such malignant conditions when diagnosing benign tumoral lesions of the spine such as meningiomas.
\end{abstract}

\section{Background and Importance}

Primary leptomeningeal melanoma (PLM) is exceedingly rare, only affecting 1 in 20 million people. ${ }^{1}$ It is one of several known melanocytic lesions in the central nervous system
(CNS). Since its initial description by Virchow in $1859, \sim 200$ cases have been reported in the literature. ${ }^{2,3}$ PLM is highly malignant and has been described both as a solid and/or diffusely growing tumor. ${ }^{3-5}$ The differential diagnosis of solid leptomeningeal lesions includes benign tumors (e.g., received

December 19, 2013

accepted

January 13, 2014

published online

May 12, 2012
DOI http://dx.doi.org/

10.1055/s-0034-1372474. ISSN 2193-6358. (c) 2014 Georg Thieme Verlag KG
Stuttgart . New York

License terms

(요 (1) $\Theta \circledast$ 

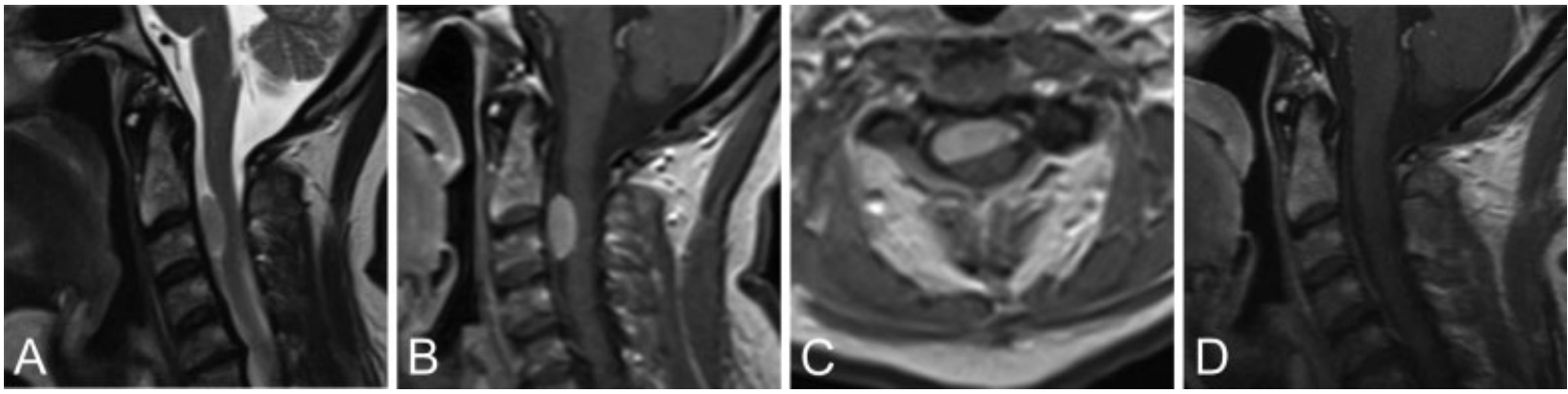

Fig. 1 (A-C) Preoperative magnetic resonance imaging (MRI) showed an intradural extramedullary solid mass anterior to the C2-C3 spinal cord causing spinal cord compression. (D) Postoperative MRI showed no residual tumor or contrast enhancement.

melanocytoma and meningioma) that can be mimicked by PLM. ${ }^{5-13}$ Meningioma is one of the most common neurosurgical conditions, constituting $>20 \%$ of primary brain tumors. $^{6}$

Spinal PLM represents both diagnostic and management challenges. ${ }^{14,15}$ We present the rare case of PLM in the ventral cervical spine, and then discuss the diagnosis and management of this lesion as a mimicker of meningioma.

\section{Clinical Presentation}

\section{Clinical Summary}

A patient in her sixth decade presented with 6 months of intermittent dizziness, slight weakness of the lower extremities, and foot paraesthesias, as well as 4 months of urge incontinence. Apart from essential hypertension, her previous medical history was negative. Physical examination revealed bilateral upper and lower extremity hyperreflexia without obvious paresis, cerebellar signs, or oculovestibular abnormalities. Magnetic resonance imaging (MRI) detected a dural-based extramedullary solid mass ventral to the $\mathrm{C2}-\mathrm{C} 3$ spinal cord causing spinal cord compression without cord signal changes (-Fig. 1A-C). The tumor appeared isointense on T1- and hyperintense on T2-weighted images with homogeneous contrast enhancement, characteristic of meningioma. Communicating hydrocephalus was also noted. Surgical resection was planned for a suspected ventral spine meningioma.

\section{Operation and Postoperative Course}

A right-sided posterolateral approach was performed to expose the lesion. Intraoperative microscopic inspection revealed numerous black spots littering the surface of the dura; the tumor itself was yellow in appearance and had a soft consistency (-Fig. 2). Gross total resection was achieved, and postoperative MRI displayed no residual tumor or contrast enhancement ( - Fig. 1D). Postoperatively, the patient's symptoms resolved completely, and she had an uncomplicated hospital course. Pathologic analysis of the specimen revealed a malignant melanin-containing tumor, which led to a thorough organ system evaluation to find the primary lesion. No primary site was found, so a diagnosis of primary leptomeningeal melanoma was made and the patient subsequently received interferon therapy. Two years postoperatively, no local or systemic recurrence of the tumor has been identified.

\section{Discussion}

\section{Clinical Evaluation}

PLM is not associated with specific clinical symptoms. ${ }^{16,17}$ In the present case, the patient's main complaints were due to tumor location and size, as others have also documented. ${ }^{2,18}$
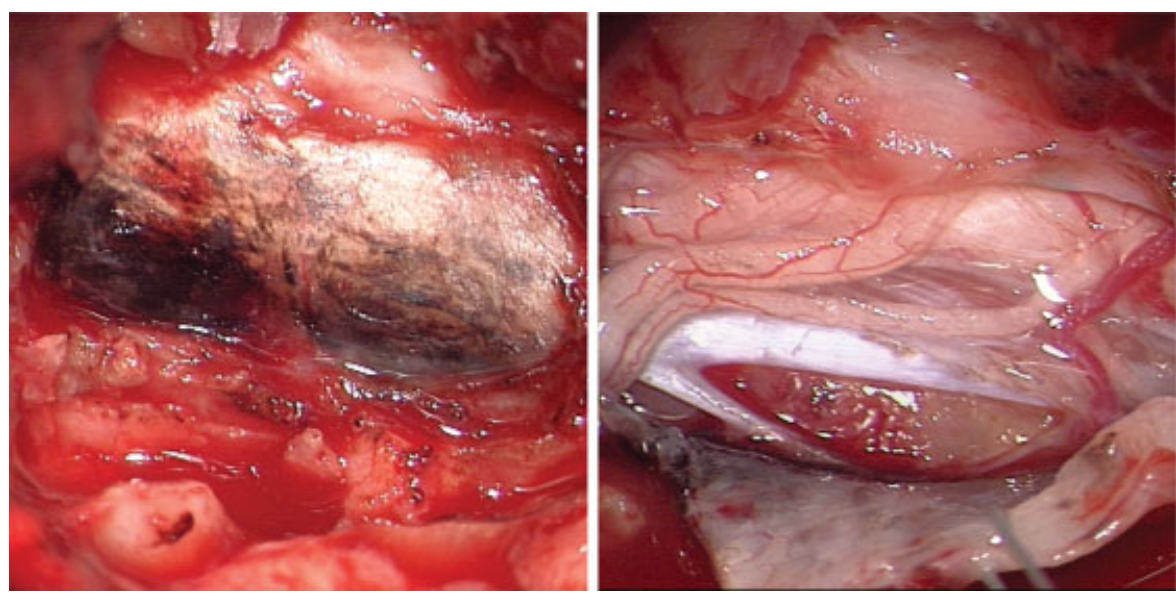

Fig. 2 (A) Intraoperatively the dura was littered with black spots. (B) The tumor itself was yellow with a soft consistency. 
Communicating hydrocephalus with associated intracranial hypertension seem to be common clinical features of melanocytic lesions in the CNS. ${ }^{2,3,9,15,19-22}$ Although the cause remains unclear, intracranial hypertension with communicating hydrocephalus can be the first and only clinical sign of PLM. ${ }^{15,23}$ Communicating hydrocephalus in the setting of diffuse leptomeningeal melanosis can be explained by features of poor CSF resorption. ${ }^{24-27}$ However, there is little evidence to explain the observed communicating hydrocephalus in solid leptomeningeal melanocytic lesions, apart perhaps from locally disrupted CSF circulation. ${ }^{23,28,29}$ The observed communicating hydrocephalus in this patient was treated by primary tumor resection; we did not measure the intracranial pressure. In $25 \%$ of all cases, PLM is seen in conjunction with neurocutaneous melanosis (i.e., large congenital nevi) that may hint at PLM. ${ }^{20,22,26,30-35}$ Similar to meningioma, the peak age of onset for PLM is in the fifth decade. $^{2}$

\section{Radiology}

The radiographic diagnosis of PLM can be problematic. ${ }^{9,14,17,18,27,33,36,37}$ The paramagnetic properties of melanin create hyperintense signal on T1-weighted images and isointense signal on T2-weighted images, which can be interpreted as intratumoral hemorrhage. ${ }^{2,3,15,17,27}$ PLM and other melanocytic lesions tend to enhance avidly with gadolinium. ${ }^{27}$ The present case is radiographically atypical for PLM in that its MRI characteristics more resemble the appearance of amelanotic melanoma without a hemorrhagic component (T1 isointense and T2 hyperintense), which is similar to the appearance of meningioma. ${ }^{7,10,18}$ The lack of melanin signal on the MRI in this case may be explained by the atypical gross appearance of the tumor (i.e., yellow with black spots on the dura as opposed to a deep brown or black-appearing mass, due to high levels of melanin). 2,18,27 The observed PLM was indistinguishable from a meningioma on MRI. Computed tomography imaging is not suitable for distinguishing between PLM and benign lesions like meningioma. 3,9,10,33 ${ }^{99 m}$ Tc-sestamibi (MIBI)-single-photon emission computed tomography (SPECT) or ${ }^{99 \mathrm{~m}} \mathrm{Tc}$-hexamethylproyleneamine oxime (HMPAO)-SPECT may be useful adjuncts to MR imaging due to increased uptake in malignancies such as PLM compared with normal brain tissue. ${ }^{17,27,33}$ Fluorodeoxyglucose (FDG)-positron emission tomography (PET) can also be helpful when trying to differentiate between metastatic melanoma and PLM. ${ }^{7}$ Clearly, suspicion of an unusual lesion on MRI or the presence of cutaneous stigmata such as giant nevi would be the only information leading the clinician toward SPECT and PET imaging.

\section{Pathology}

Melanocytes can be found not only in skin and mucosal membranes, but also in the leptomeninges, among other locations. All melanocytes can give rise to melanocytic lesions. ${ }^{2,3,10}$ The value of cerebrospinal fluid (CSF) cytology in diagnosing PLM is questionable, especially in the early

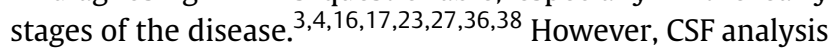
for atypical pigmented cells followed by immunohistochemical analysis with the monoclonal antibody HMB-45 in combination with S-100 protein appear more reliable. ${ }^{17,39,40}$ PLM is primarily diagnosed by histology at autopsy or by analyzing resected tissue postoperatively. $3,4,16,21,27,37,38,41$ The hallmark histologic features of PLM include positive immunohistochemical staining for HMB-45, S-100 protein, and vimentin as well as negative testing for glial fibrillary acid protein (GFAP) and epithelial membrane antigen ${ }^{42}$ that exclude a glial origin and meningioma. $2,7,9,10,23$ In the present case, histology revealed a cellular neoplasm with moderately polymorphic cells with prominent nucleoli and focal deposition of brown pigment. Scattered mitoses were detectable (-Fig. 3A). Immunohistochemically, the tumor cells showed a positive reaction with antibodies $H M B-45$, Melan- $A$, and vimentin and a negative reaction with GFAP. ${ }^{42}$ MIB-1 staining was $5 \%$ in the present case (-Fig. 3B), which provided conclusive evidence of malignancy and excluded melanocytoma, PLM's benign counterpart. It should be noted that intermediate grades of melanocytic tumors do exist. ${ }^{5,7,9,23,31,43}$ The absence of an additional tumor source in conjunction with the pathologic findings just described led to the diagnosis of a solitary PLM in this patient. ${ }^{44}$

\section{Treatment and Prognosis}

Although PLM has a better prognosis than metastatic melanoma, ${ }^{10,18}$ it is highly malignant and median survival is $<6$ months. ${ }^{3,15}$ Diffuse PLM has a worse prognosis than nodular PLM. ${ }^{17,21,36}$ Gross total resection (GTR) of solid lesions is usually performed and has been assumed to contribute to prolonged survival in many cases, although not all. 2,3,5,8,10,11,13,14,29 A possible explanation for the exceptionally poor outcomes, despite successful GTR, may be misdiagnosis of PLM in the setting of actual metastatic melanoma, where the primary tumor (for instance, on the
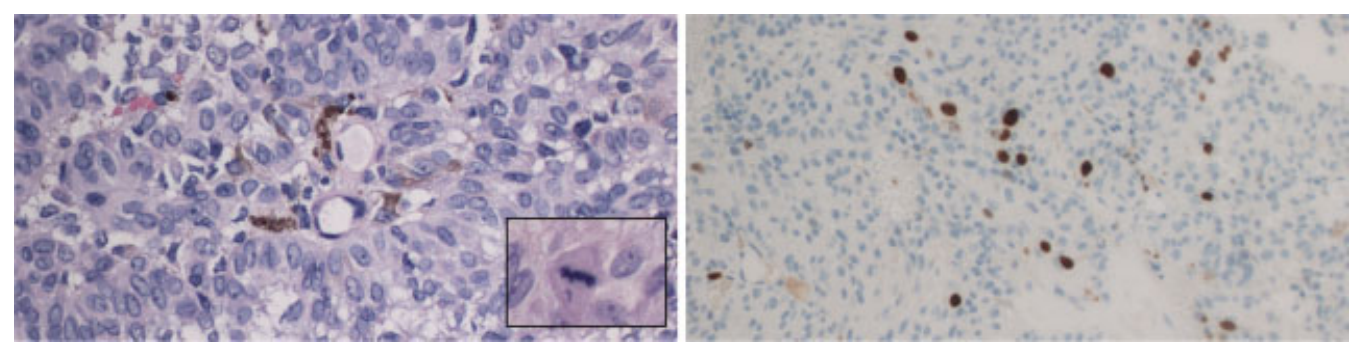

Fig. 3 (A) Histology revealed cell clusters with brown pigmented spots suggestive of a malignant melanoma. (B) MIB-1 staining was $5 \%$. 
skin) spontaneously regressed and went undetected. ${ }^{7}$ These points emphasize the need for a multimodal approach including radiotherapy and intrathecal chemotherapy as has been suggested. $3,23,31,43,45$ The patient in the present case had a GTR followed by interferon therapy. To date ( 2 years after surgery), there are no clinical or radiologic signs of tumor recurrence. We did not treat the patient's communicating hydrocephalus for two reasons: (1) the patient was symptom free after surgery, and (2) there is a risk of spreading malignant cells into the peritoneal cavity through CSF diversion with a ventriculoperitoneal shunt. ${ }^{2,31}$ We have closely monitored this patient both clinically and radiographically based on recommendations in the literature. ${ }^{43}$

\section{Conclusion}

As with most rare tumors, case reports constitute the vast majority of references to PLM. Thus no gold standard currently exists for the definitive treatment and follow-up of patients with this diagnosis. ${ }^{7,43}$ Several reasonably valuable tools are available for diagnosing PLM, namely, immunohistochemical CSF analysis, MRI, and SPECT. It is unlikely, however, that CSF analysis or SPECT would be performed if MRI results pointed to the incorrect diagnosis of meningioma. Only an increased awareness through an extensive report of each individual case can help diagnose and clarify the nature of PLM. Clinicians need to be aware of such malignant conditions when diagnosing suspected benign tumoral spine lesions such as meningiomas.

\section{Authors' Contributions}

Conception and design of the manuscript: Marx, Fleck, Manwaring, Schroeder. Acquisition of data: all authors. Analysis and interpretation of data: Marx, Fleck, Manwaring, Schroeder. Drafting of the article: Marx, Fleck, Manwaring. Critical review, revision, and approval of the final manuscript: all authors.

\section{References}

1 Helseth A, Helseth E, Unsgaard G. Primary meningeal melanoma. Acta Oncol 1989;28(1):103-104

2 Liubinas SV, Maartens N, Drummond KJ. Primary melanocytic neoplasms of the central nervous system. J Clin Neurosci 2010; 17(10):1227-1232

3 Allcutt D, Michowiz S, Weitzman S, et al. Primary leptomeningeal melanoma: an unusually aggressive tumor in childhood. Neurosurgery 1993;32(5):721-729, discussion 729

4 López-Castilla J, Díaz-Fernández F, Soult JA, Muñoz M, Barriga R. Primary leptomeningeal melanoma in a child. Pediatr Neurol 2001;24(5):390-392

5 Brat DJ, Giannini C, Scheithauer BW, Burger PC. Primary melanocytic neoplasms of the central nervous systems. Am J Surg Pathol 1999;23(7):745-754

6 Ghosal N, Dadlani R, Gupta K, Furtado SV, Hegde AS. A clinicopathological study of diagnostically challenging meningioma mimics. J Neurooncol 2012;106(2):339-352

7 Wadasadawala T, Trivedi S, Gupta T, Epari S, Jalali R. The diagnostic dilemma of primary central nervous system melanoma. J Clin Neurosci 2010;17(8):1014-1017
8 O'Brien DF, Crooks D, Mallucci C, et al. Meningeal melanocytoma. Childs Nerv Syst 2006;22(6):556-561

9 Kashiwagi N, Hirabuki N, Morino H, Taki T, Yoshida W, Nakamura $\mathrm{H}$. Primary solitary intracranial melanoma in the sylvian fissure: MR demonstration. Eur Radiol 2002;12(Suppl 3):S7-S10

10 Greco Crasto S, Soffietti R, Bradac GB, Boldorini R. Primitive cerebral melanoma: case report and review of the literature. Surg Neurol 2001;55(3):163-168; discussion 168

11 Farnsworth TA. Primary cerebral malignant melanoma: an unusual cause of dyspraxia. Int J Clin Pract 1998;52(6):445-446

12 O'Brien TF, Moran M, Miller JH, Hensley SD. Meningeal melanocytoma. An uncommon diagnostic pitfall in surgical neuropathology. Arch Pathol Lab Med 1995;119(6):542-546

13 Barut S. Primary leptomeningeal melanoma simulating a meningioma. Neurosurg Rev 1995;18(2):143-147

14 Mondot L, Almairac F, Vandenbos F, Fontaine D, Frenay M, Chanalet S. Primitive cerebral melanoma: a diagnostic and management challenge. About 2 cases. J Neuroradiol 2012;39(3):200-204

15 Demir MK, Aker FV, Akinci O, Ozgültekin A. Case 134: primary leptomeningeal melanomatosis. Radiology 2008;247(3):905-909

16 Pirini MG, Mascalchi M, Salvi F, et al. Primary diffuse meningeal melanomatosis: radiologic-pathologic correlation. AJNR Am J Neuroradiol 2003;24(1):115-118

17 Tosaka M, Tamura M, Oriuchi N, et al. Cerebrospinal fluid immunocytochemical analysis and neuroimaging in the diagnosis of primary leptomeningeal melanoma. Case report. J Neurosurg 2001;94(3):528-532

18 Unal B, Castillo M. MRI features of a primary thoracic epidural melanoma: a case report. Clin Imaging 2007;31(4):273-275

19 Arias M, Alberte-Woodward M, Arias S, Dapena D, Prieto A, SuárezPeñaranda JM. Primary malignant meningeal melanomatosis: a clinical, radiological and pathologic case study. Acta Neurol Belg 2011;111(3):228-231

20 Zhang N, Liu ZJ, Li G, et al. Clinical and pathologic features of melanocytic lesion of the central nervous system. [in Chinese]. Zhong Nan Da Xue Xue Bao Yi Xue Ban 2007;32(4):713-717

21 Bobba R, Arsura E. Cognitive decline in an elderly hospitalized patient with primary leptomeningeal melanomatosis. South Med J 2004;97(11):1118-1120

22 Chang CS, Hsieh PF, Chia LG, et al. Leptomeningeal malignant melanoma arising in neurocutaneous melanocytosis: a case report. Zhonghua Yi Xue Za Zhi (Taipei) 1997;60(6):316-320

23 Kounin GK, Romansky KV, Traykov LD, Shotekov PM, Stoilova DZ. Primary spinal melanoma with bilateral papilledema. Clin Neurol Neurosurg 2005;107(6):525-527

24 Bocquillon P, Berteloot AS, Maurage CA, Mackowiak-Cordoliani MA, Pasquier F, Bombois S. Primitive leptomeningeal malignant melanoma: a rare etiology of pachymeningitis and leptomeningitis. [in French]. Rev Neurol (Paris) 2010;166(11):927-930

25 Bajer-Czajkowska A, Nowacki P. Primary diffuse meningeal melanomatosis. Case report. [in Polish]. Neurol Neurochir Pol 2007; 41(1):82-88

26 de Andrade DO, Dravet C, Raybaud C, Broglin D, Laguitton V, Girard $\mathrm{N}$. An unusual case of neurocutaneous melanosis. Epileptic Disord 2004;6(3):145-152

27 Sagiuchi T, Ishii K, Utsuki S, et al. Increased uptake of technetium99m-hexamethylpropyleneamine oxime related to primary leptomeningeal melanoma. AJNR Am J Neuroradiol 2002;23(8): 1404-1406

28 Azimi P, Mohmmadi HR, Refiezadeh M. Primary pineal melanoma presenting with leptomeningeal spreading in a 22-year-old woman: a case report. J Med Case Reports 2012;6(1):165

29 Yamane K, Shima T, Okada Y, et al. Primary pineal melanoma with long-term survival: case report. Surg Neurol 1994;42(5):433-437

30 Furtado S, Furtado SV, Ghosal N, Hegde AS. Fatal leptomeningeal melanoma in neurocutaneous melanosis. Pediatr Dermatol 2012; 29(3):358-361 
31 Shinno K, Nagahiro S, Uno M, et al. Neurocutaneous melanosis associated with malignant leptomeningeal melanoma in an adult: clinical significance of 5-S-cysteinyldopa in the cerebrospinal fluid -case report. Neurol Med Chir (Tokyo) 2003;43(12):619-625

32 Oka H, Kameya T, Hata T, Kawano N, Fujii K, Yada K. Leptomeningeal melanomatosis with multiple cutaneous pigmented nevi: tumor cell proliferation and malignant transformation in an autopsy case. J Neurooncol 1999;44(1):41-45

33 Soler C, Beauchesne P, Moncet M, et al. 99mTc-MIBI uptake in a primitive leptomeningeal melanoma. Eur J Dermatol 1998;8(3): 169-172

34 Kadonaga JN, Frieden IJ. Neurocutaneous melanosis: definition and review of the literature. J Am Acad Dermatol 1991;24(5 Pt 1): $747-755$

35 Sawamura Y, Abe H, Murai H, Tashiro K, Doi S. An autopsy case of neurocutaneous melanosis associated with intracerebral malignant melanoma. [in Japanese]. No To Shinkei 1987;39(8): 789-795

36 Rosenthal G, Gomori JM, Tobias S, Diment J, Shoshan Y. Unusual cases involving the CNS and nasal sinuses: Case 1. Primary leptomeningeal melanoma. J Clin Oncol 2003;21(20):3875-3877

37 Akai T, Kuwayama N, Ogiichi T, Kurimoto M, Endo S, Takaku A. Leptomeningeal melanoma associated with straight sinus thrombosis-case report. Neurol Med Chir (Tokyo) 1997;37(10): 757-761
38 Monsó G, Vernet A, Cusí V, Mateo M, Vilaseca MA. Primary leptomeningeal melanomatosis in a 6-year-old child. [in Spanish]. Rev Neurol 1996;24(125):87-90

39 Bamborschke S, Ebhardt G, Szelies-Stock B, Dreesbach HA, Heiss WD. Review and case report: primary melanoblastosis of the leptomeninges. Clin Neuropathol 1985;4(2):47-55

40 Aichner F, Schuler G. Primary leptomeningeal melanoma. Diagnosis by ultrastructural cytology of cerebrospinal fluid and cranial computed tomography. Cancer 1982;50(9):1751-1756

41 Fish LA, Friedman DI, Sadun AA. Progressive cranial polyneuropathy caused by primary central nervous system melanoma. J Clin Neuroophthalmol 1990;10(1):41-44

42 Asi-Bautista MC, Heidemann SM, Meert KL, Canady AI, Sarnaik AP. Tumor necrosis factor-alpha, interleukin- 1 beta, and interleukin- 6 concentrations in cerebrospinal fluid predict ventriculoperitoneal shunt infection. Crit Care Med 1997;25(10):1713-1716

43 Eskandari R, Schmidt MH. Intramedullary spinal melanocytoma. Rare Tumors 2010;2(2):e24

44 Hayward RD. Malignant melanoma and the central nervous system. A guide for classification based on the clinical findings. J Neurol Neurosurg Psychiatry 1976;39(6):526-530

45 Fathallah-Shaykh HM, Zimmerman C, Morgan H, Rushing E, Schold SC Jr, Unwin DH. Response of primary leptomeningeal melanoma to intrathecal recombinant interleukin-2. A case report. Cancer 1996;77(8):1544-1550 